\title{
An activated mutant BRAF kinase domain is sufficient to induce pilocytic astrocytoma in mice
}

\author{
Jan Gronych, ${ }^{1}$ Andrey Korshunov, ${ }^{2}$ Josephine Bageritz, ${ }^{1}$ Till Milde,,$^{3,4}$ \\ Manfred Jugold, 5 Dolores Hambardzumyan, 6 Marc Remke, ${ }^{1,4}$ Christian Hartmann, ${ }^{2}$ \\ Hendrik Witt,, ${ }^{1,4}$ David T.W. Jones, ${ }^{1}$ Olaf Witt,, ${ }^{3,4}$ Sabine Heiland, ${ }^{7}$ Martin Bendszus, ${ }^{7}$ \\ Eric C. Holland, ${ }^{6}$ Stefan Pfister, ${ }^{1,4}$ and Peter Lichter ${ }^{1}$
}

\begin{abstract}
${ }^{1}$ Division Molecular Genetics (B060), German Cancer Research Center, Heidelberg, Germany.
${ }^{2}$ Clinical Cooperation Unit Neuropathology (G380), German Cancer Research Center, and Department of Neuropathology, Institute of Pathology, Ruprecht-Karls-University, Heidelberg, Germany. ${ }^{3}$ Clinical Cooperation Unit Pediatric Oncology (G340), German Cancer Research Center, Heidelberg, Germany. ${ }^{4}$ Department of Pediatric Hematology and Oncology, University Hospital Heidelberg, Heidelberg, Germany. ${ }^{5}$ Division Medical Physics in Radiology, German Cancer Research Center, Heidelberg, Germany. ${ }^{6}$ Cancer Biology and Genetics, Memorial Sloan-Kettering Cancer Center, New York, New York, USA. 'Department of Neuroradiology, University Hospital Heidelberg, Heidelberg, Germany.
\end{abstract}

\begin{abstract}
Pilocytic astrocytoma (PA) is the most common type of primary brain tumor in children and the second most frequent cancer in childhood. Children with incompletely resected PA represent a clinically challenging patient cohort for whom conventional adjuvant therapies are only moderately effective. This has produced high clinical demand for testing of new molecularly targeted treatments. However, the development of new therapeutics for PA has been hampered by the lack of an adequate in vivo tumor model. Recent studies have identified activation of MAPK signaling, mainly by oncogenic $B R A F$ activation, as a hallmark genetic event in the pathogenesis of human PA. Using in vivo retroviral somatic gene transfer into mouse neural progenitor cells, we have shown here that ectopic expression of the activated $B R A F$ kinase domain is sufficient to induce PA in mice. Further in vitro analyses demonstrated that overexpression of activated $B R A F$ led to increased proliferation of primary mouse astrocytes that could be inhibited by treatment with the kinase inhibitor sorafenib. Our in vivo model for PA shows that the activated $B R A F$ kinase domain is sufficient to induce PA and highlights its role as a potential therapeutic target.
\end{abstract}

\section{Introduction}

Pilocytic astrocytoma $(\mathrm{PA})$ is the most common primary brain tumor in children. This WHO grade I neoplasm characteristically displays noninfiltrative growth and shows benign biological behavior that translates into a remarkably high 10-year overall survival rate of greater than $90 \%$ upon gross total resection (1). Nevertheless, up to $20 \%$ of patients may not be cured by surgery alone (2). Because of the slow-growing nature of PA, complete responses to adjuvant radiation and chemotherapy are rare, and most tumors eventually progress after a stabilization period of typically more than 3 years. Thus, there is demand for novel targeted therapeutic options (3).

Recent studies suggest that most PAs feature constitutive activation of the MAPK signaling cascade as a molecular hallmark. Thus, PA may be a prototypic single-pathway disease, with MAPK signaling constituting the critically important molecular target for future therapeutic intervention. Previously, we identified tandem duplications or activating mutations (V600E) of the proto-oncogene BRAF at $7 \mathrm{q} 34$ as by far the most prevalent genetic mechanism leading to constitutive MAPK pathway activation, occurring in more than 50\% of sporadic PAs (4). Subsequent studies confirmed similar or even higher frequencies of BRAF tandem duplications and activating mutations in PAs, with V600E being the most frequent, and showed that the duplication leads to formation of fusion genes of $B R A F(5-9)$. The unifying molecular feature of all RAF fusion products identi-

Conflict of interest: The authors have declared that no conflict of interest exists. Citation for this article: J Clin Invest. 2011;121(4):1344-1348. doi:10.1172/JCI44656. fied to date is that the RAF kinase domain alone, upon loss of the autoinhibitory $\mathrm{N}$ terminus, has the potential to drive proliferation via constitutive MAPK activation. Hence, it is commonly assumed that RAF activation, either by fusion or by mutation, constitutes a critically important genetic event in PA tumorigenesis.

Approaches based on constitutive MAPK activation were pursued to model human brain tumors in mice (10-12). However, generation of an adequate model for PA has been hampered by the low proliferative character of these tumors and a lack of knowledge about the molecular etiology.

To assess the oncogenic potential of $B R A F$ in the formation of astrocytic tumors, we used the replication-competent avian leukosis virus with splice acceptor/Tv-a (RCAS/Tv-a) system for retroviral gene transfer $(13,14)$. Using this system, we introduced different BRAF constructs into nestin-expressing neural progenitor cells of newborn mice. We show that transgenic expression of the activated BRAF kinase domain was sufficient to induce PA formation in mice, with tumors closely recapitulating the clinical and histological features of human PA.

\section{Results and Discussion}

Generation of MAPK-activating RCAS-BRAF variants. To assess the role of $B R A F$ in brain tumor etiology, we cloned a variety of $B R A F$ constructs the full-length gene (BRAF WT FL), the full-length gene carrying the V600E mutation (BRAF VE FL), the WT kinase domain spanning exons 9-18 of WT BRAF (BRAF WT kin), and the V600E mutated $B R A F$ kinase domain (BRAF VE kin) - into the RCASBP(A) vector 
A

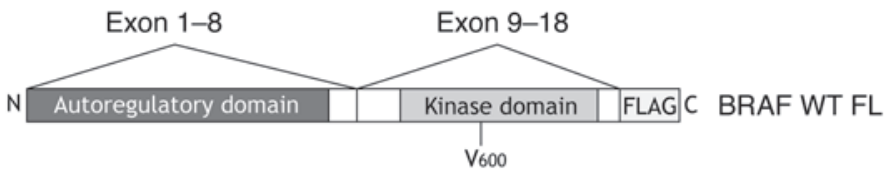

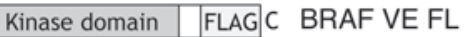
E600

Kinase domain $\quad$ FLAG C BRAF WT kin $\mathrm{V}_{600}$

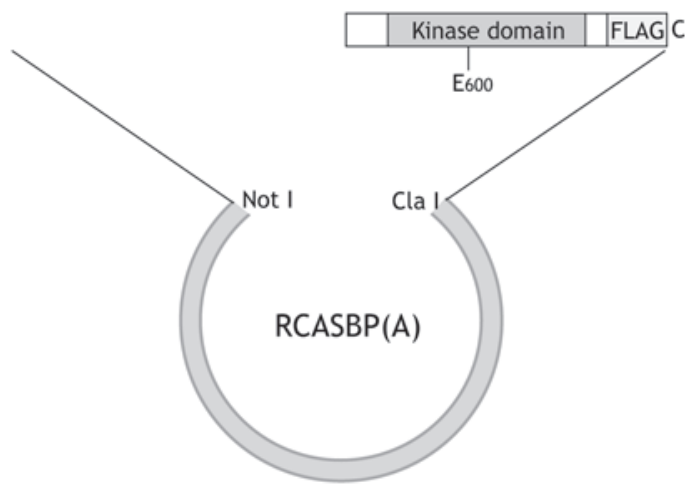

B
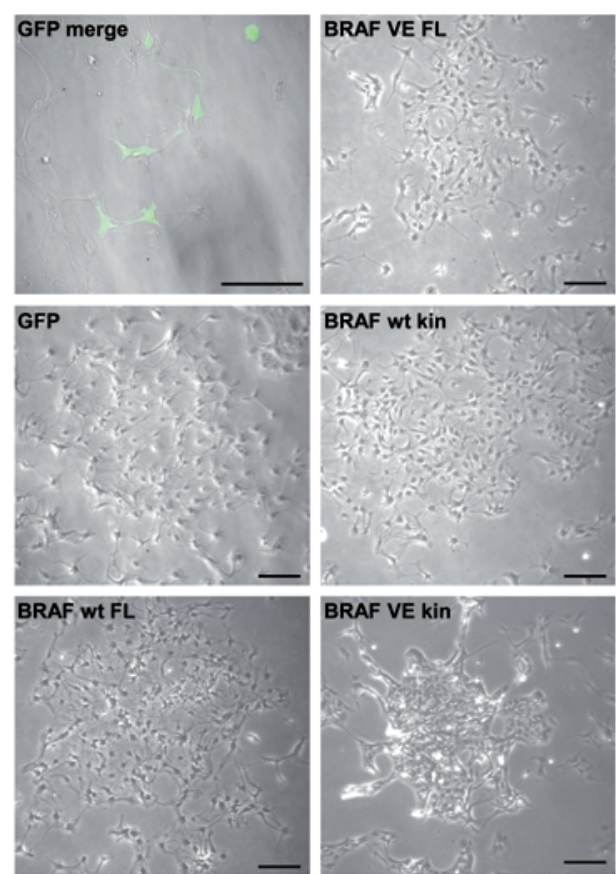

\section{Figure 1}

BRAF variants induce MAPK activation. (A) Different BRAF constructs used for RCAS-mediated gene delivery. Truncated variants containing the kinase domain corresponded to exons 9-18 of the human BRAF gene. The V600E mutation is indicated. (B) Phase-contrast microscopy demonstrated altered cell morphology and focal growth of cells transduced with BRAF VE kin compared with control and other BRAF constructs. Scale bars: $100 \mu \mathrm{m}$.

(Figure 1A). Transduction of primary Ntv-a astrocytes with these different constructs and GFP as a control in vitro revealed morphological alterations and focal growth induced by overexpression of BRAF VE kin (Figure 1B). Cells expressing all other constructs displayed a morphology similar to the GFP control.

BRAF VE kin induces tumors in Ntv-a mice. For expression of BRAF variants in neural progenitor cells in vivo, we injected virus-producing DF-1 cells into hemispheres or brainstem of neonatal Ntv-a mice for each of the 4 variants (Table 1). In the first cohort, 7 of 39 animals died as a result of encephalitis during the first weeks after injection. At 17 weeks after inoculation, all surviving mice were still clinically asymptomatic. These animals were then sacrificed, and presence of a tumor was detected upon histopathologic evaluation only in animals overexpressing BRAF VE kin. In a second cohort, BRAF VE kin was injected into the cerebral hemispheres. Using highfield MRI, unilateral hemispheric hyperintensity in T1-weighted images after application of contrast agent was detected (Figure 2A). Of note, this radiologic finding resembled the MRI appearance of human PAs, which are frequently contrast enhancing. Postmortem histopathologic analysis confirmed the presence of glial tumors based on GFAP immunoreactivity. Furthermore, activated MAPK signaling, as assessed by immunohistochemical staining for Erk phosphorylation, overlapped with the contrast enhancement seen in the MRI and with GFAP staining. FLAG-tagged BRAF transgene was also detected in the neoplastic region, but exhibited more diffuse staining (Figure 2A). Strikingly, the relatively slow tumor growth seen upon RCAS-mediated oncogene induction again recapitulated the biological behavior of human PAs. Tumor-bearing animals were clinically asymptomatic until at least 4 months after induction; in contrast, animals subjected to tumor induction with RCAS-PDGF survive no longer than 3 months in the same system (15).

Tumor incidence and histology. Histopathological analysis revealed a total of 21 neoplasias in the cerebral hemispheres $(n=17)$ and in the brainstem $(n=4)$. All of these lesions were found upon overexpression of BRAF VE kin, with an incidence of $91 \%$ (21 of 23 ; Table 1 ). With all remaining constructs, no tumor development was observed up to 4 months after injection. This confirmed the findings of an earlier study, in which overexpression of full-length WT BRAF or the full-length V600E variant alone did not result in tumor formation after 3 months, whereas overexpression of BRAF VE FL resulted in high-grade astrocytic tumors only on an Ink4/Arf-deficient background (16). This, in turn, is consistent with considerations on the role of Ink4/Arf in Ras-driven mouse models in the literature (17).

In our study, BRAF VE kin-induced cerebral tumors exhibited more extensive growth within the hemisphere along the lateral ventricle, compared with the more focal appearance of brainstem

\section{Table 1}

Incidence and histologic grade of tumors induced by $B R A F$ variants

$\begin{array}{lcc}\text { Construct } & \text { Incidence } & \text { Histology } \\ \text { BRAF WT FL } & 0 / 10(0 \%) & - \\ \text { BRAF VE FL } & 0 / 10(0 \%) & - \\ \text { BRAF WT kin } & 0 / 10(0 \%) & - \\ \text { BRAF VE kin } & 21 / 23(91 \%) & \text { Pilocytic astrocytoma }\end{array}$


A

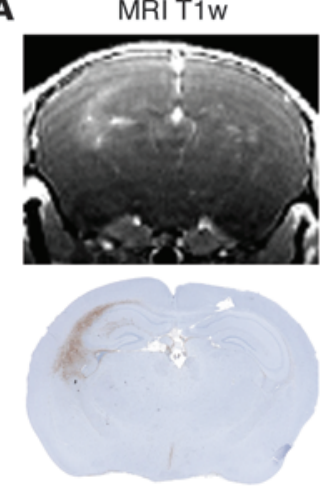

GFAP

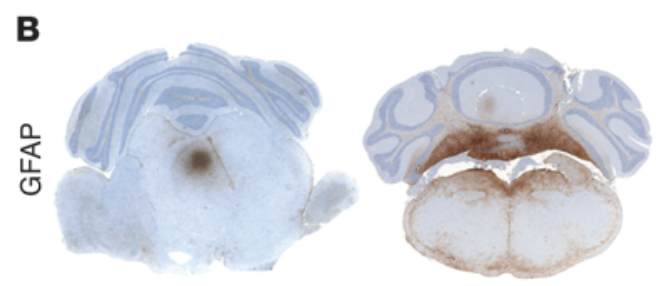

pErk
C
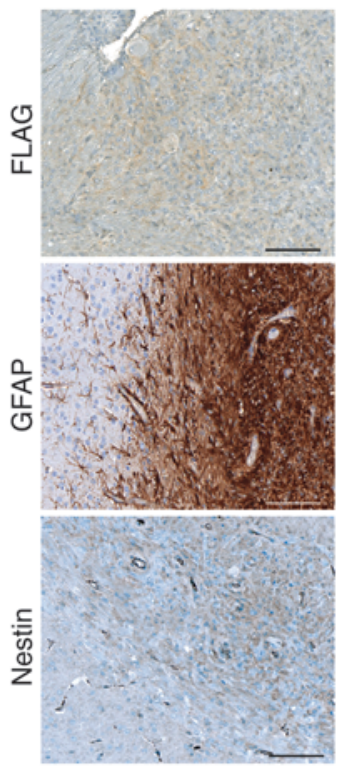

D
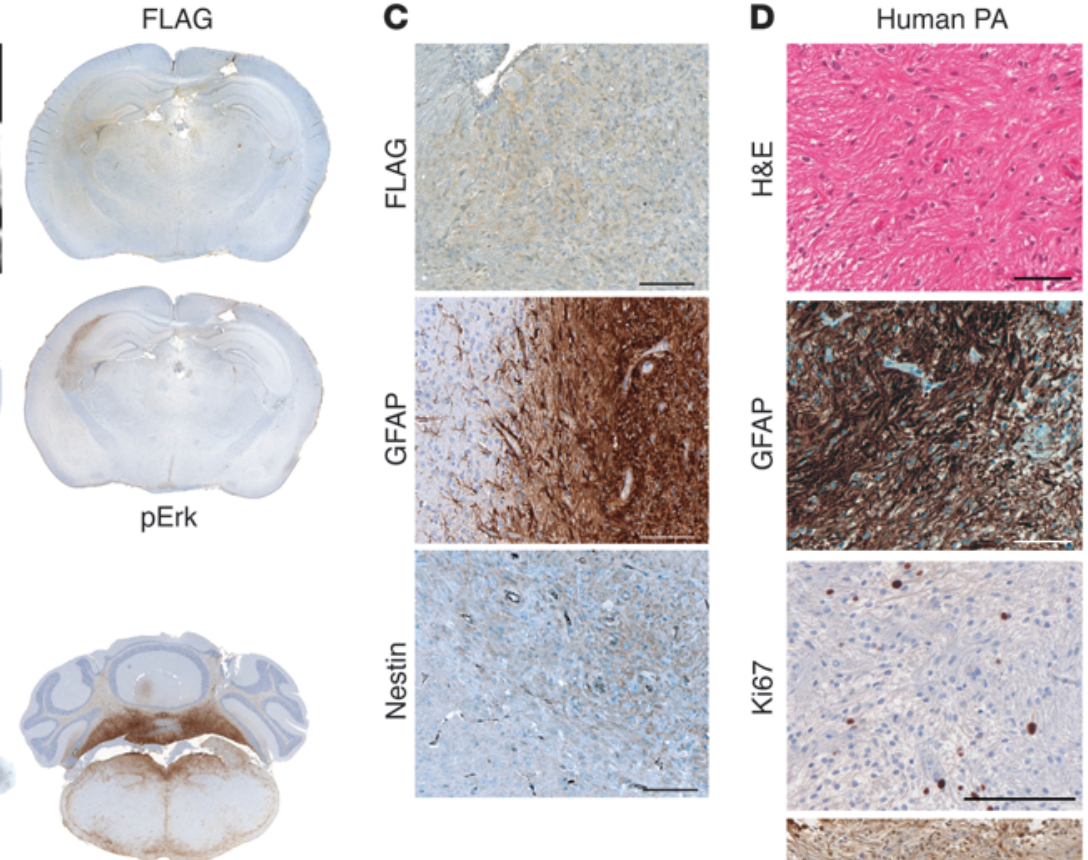
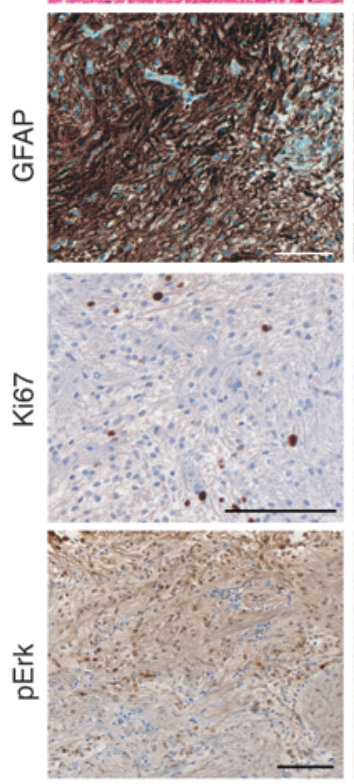

Murine PA
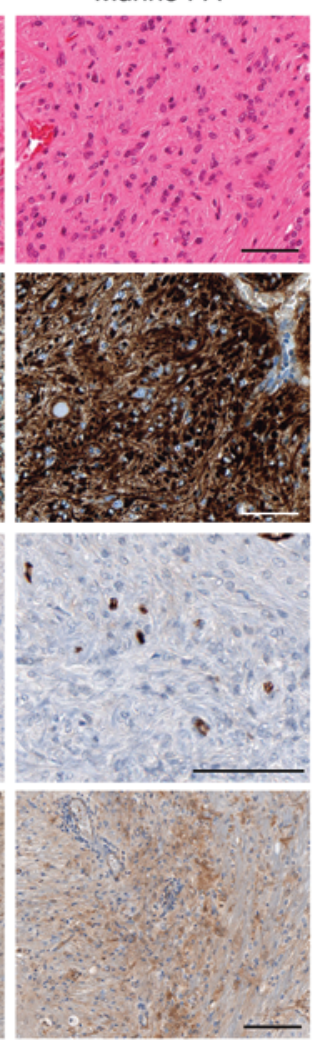

Figure 2

MRI and tumor histology. (A) Hemispheric contrast-enhancing tumor induced with BRAF VE kin, as observed in T1-weighted MRI and coronal brain sections, showed overlapping expression of GFAP, the BRAF VE kin transgene (FLAG-tag), and Erk phosphorylation after immunohistochemical staining. (B) Tumors were also induced in the brainstem and cerebellum after expression of BRAF VE kin. (C) Higher-magnification views show the presence of transgene (FLAG), tumor delineation from normal tissue, and stronger GFAP staining of tumor cells compared with adjacent normal reactive astrocytes. Nestin staining of tumor cells was low compared to endothelial cells in neoplastic and normal tissue that exhibited a strong immunoreactivity. (D) Histological comparison between human PA and murine BRAF VE kin-induced PA. H\&E-stained sections showed histological features of PA, such as piloid tumor cells, eosinophilic Rosenthal fibers, and moderate cellularity. GFAP staining revealed strong immunoreactivity with a tight network of elongated processes and protein droplets characteristic for PA. The low proliferation index of both tumors was represented by Ki67 staining. Tumors displayed Erk phosphorylation in both cases, indicating constitutive MAPK activation. Scale bars: $100 \mu \mathrm{m}$.

tumors (Figure 2, A and B). In one of the cerebral tumors, neoplastic tissue was also found in the cerebellum (Figure 2B). In all cases, neoplastic tissue showed the presence of the transgene, and tumors were well-delineated from healthy tissue, as shown by FLAG and GFAP staining, respectively (Figure 2C). Staining of nestin, a marker for neural precursors, showed weak to negative immunoreactivity (Figure 2C). Histopathological analysis of the tumors revealed characteristic morphological patterns closely resembling human PA (Figure 2D). Tumor cells showed typical biphasic, piloid patterns and a predominantly elongated shape with round or oval, frequently pale nuclei. Eosinophilic elongated structures resembling Rosenthal fibers and eosinophilic granular bodies, so-called protein droplets, were interspersed. Blood vessels were relatively scarce. Endothelial proliferation, microcystic degeneration, or regions with oligodendroglioma-like cells were not observed. All tumors showed strong immunopositivity for GFAP, which demonstrated in the majority of tumor cells the presence of bipolar long processes forming a tight network in the intercellular spaces. Intensity of GFAP immunostaining was noticeably stronger than in reactive astrocytes from surrounding brain tissue (Figure 2C). The low pro- liferative index, as assessed by Ki67 immunohistochemistry, and the clear phospho-Erk positivity were again reminiscent of human PA (Figure 2D). These observations underline the similarity between the induced murine tumors and their human counterparts.

$M A P K$ activation and proliferation after BRAF overexpression and sorafenib treatment. To further investigate the effects of BRAF overexpression in vitro, we isolated primary neurospheres from Ntv-a mice and transduced them with the 4 different RCAS-BRAF constructs or RCAS-GFP. Expression of BRAF variants was confirmed on protein and RNA levels (Figure 3A). Interestingly, although successful viral transduction was demonstrated by quantitative RT-PCR, protein levels of BRAF VE FL were considerably reduced and only slightly above endogenous levels compared with all other variants. This is in line with a previous report showing that intrinsic stability of the V600E form of BRAF is reduced compared with the WT protein (18). In spite of this, increased MAPK activation was detected, as reflected by phosphorylation of Erk $1 / 2$ compared with the control (Figure 3A). BRAF VE kin, although showing lower RNA abundance, was present in higher protein amounts and led to clearly increased MAPK activation. In both cases, Erk phosphoryla- 
A
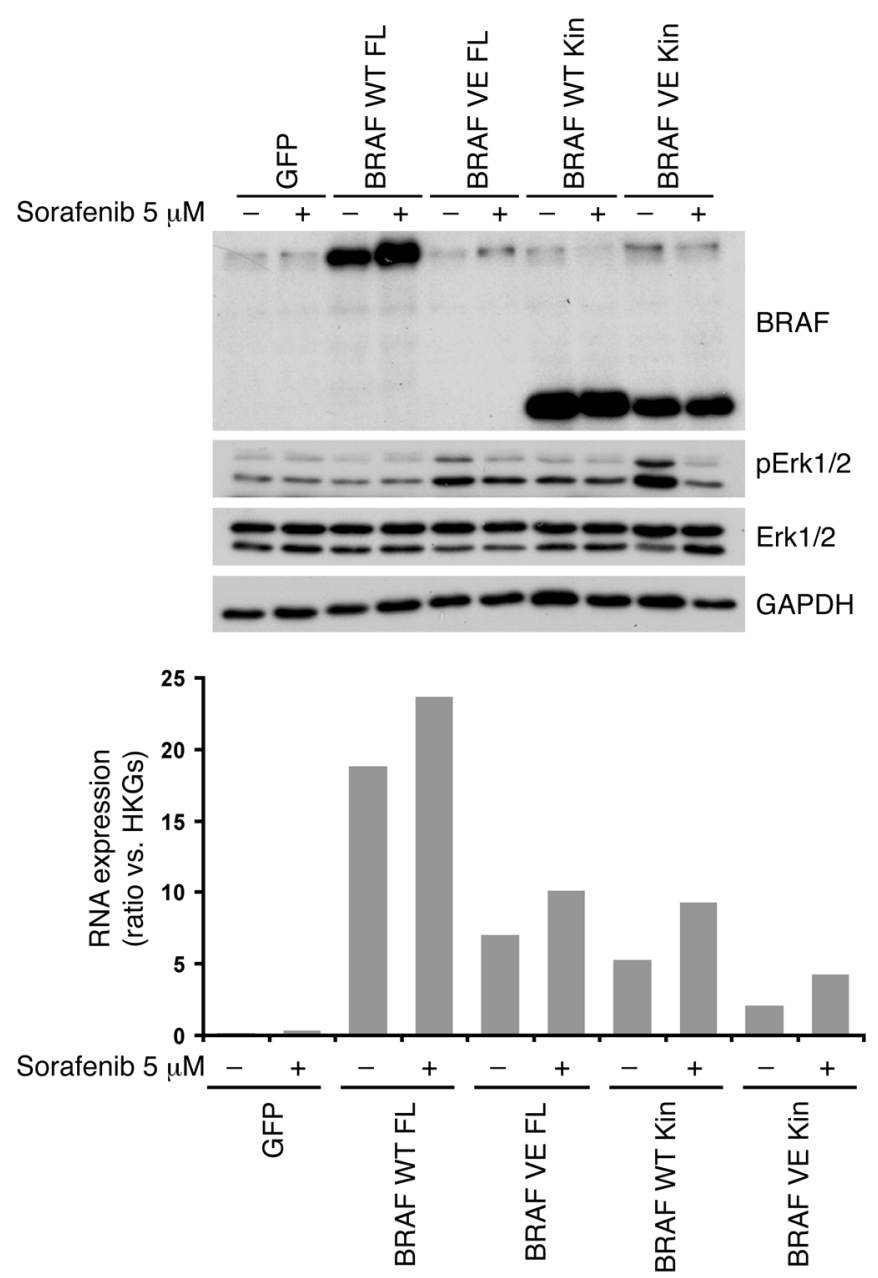

B
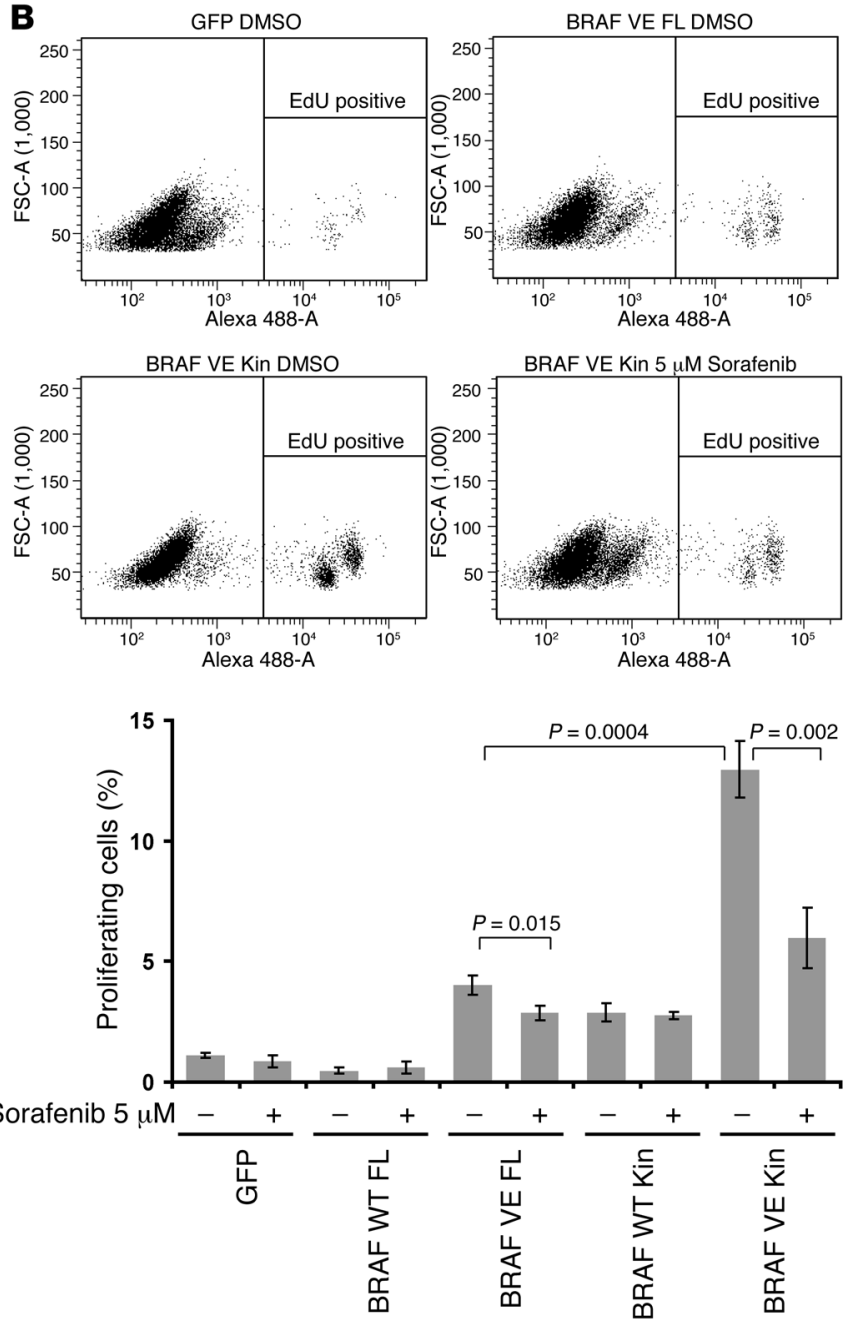

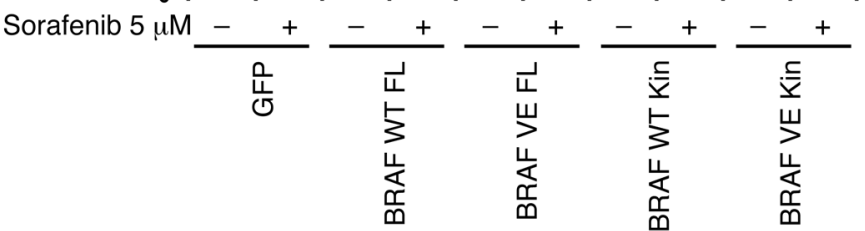

Figure 3

Transformation and sorafenib treatment of primary astrocytes in vitro. (A) Primary Ntv-a astrocytes were transduced in vitro with BRAF variants and GFP as a control. BRAF VE FL protein was considerably reduced, whereas all other variants showed high expression after transduction. Stronger activation of Erk phosphorylation in BRAF VE kin than in BRAF VE FL was decreased to control levels upon treatment with $5 \mu \mathrm{M}$ sorafenib for 6 hours. DMSO was used as vehicle control. All other variants did not show increased Erk phosphorylation. BRAF transcript abundance showed high variation among constructs depending on transduction efficiency (normalized to 3 housekeeping genes [HKGs]). (B) Representative FACS plots from EdU incorporation assays of astrocytes transduced and treated as indicated, and quantification of the proliferating fraction for all BRAF variants and GFP treated with $5 \mu \mathrm{M}$ sorafenib or DMSO control. Proliferation and RNA and protein expression were assayed from the same experiment. FSC, forward scatter. Data are mean \pm SD of 3 technical replicates.

tion was abrogated by treatment with $5 \mu \mathrm{M}$ sorafenib (Figure 3A). The WT variants did not induce detectable MAPK activation in this context. Therefore, absence of tumors after overexpression of BRAF VE FL could be attributed to the reduced protein abundance of this variant, whereby its MAPK activation does not suffice for neoplastic transformation without further oncogenic hits.

To assess the effect of the different BRAF constructs on proliferation, transduced cells were incubated with EdU in serum-containing medium for 48 hours (Figure 3B). GFP-transduced cells showed a very low proliferation rate of $1.1 \% \pm 0.1 \%$. Whereas expression of BRAF VE FL and BRAF WT kin increased the proliferating fraction to $4.0 \% \pm 0.4 \%$ and $2.9 \% \pm 0.4 \%$, respectively, BRAFVEkinled toanincrease to $13.0 \% \pm 1.2 \%$. Proproliferative effects of the V600E constructs were significantly abrogated by treating the cells with $5 \mu \mathrm{M}$ of the kinase inhibitor sorafenib (Figure 3B), which highlights the potential of MAPK inhibi- tors for clinical use. Since in human PA, oncogenic BRAF activation by fusion genes that lack activating point mutations is much more frequent than that by the V600E mutation itself, it will be highly interesting and relevant for preclinical testing of targeted drugs to model tumor induction by BRAF fusion genes in the future.

In conclusion, we provide in vivo evidence that BRAF activation is sufficient to generate PA in mice and demonstrate that the histopathological and biological characteristics of induced murine tumors are highly reminiscent of human PA. Thus, we have generated a mouse model that allows for comprehensive preclinical testing in a system that closely resembles the situation in humans.

\section{Methods}

Vector construction and in vivo infection. C-terminally FLAG-tagged BRAF variants were amplified using primers containing ClaI and NotI restriction sites 
(NotI_BRAF-Kin380, GGGGGCGGCCGCACCATGGACTTGATTAGAGACCAAG; ClaI_BRAF-Kin reverse, GGGGATCGATCTACTTATCGTCGTCATCCTTGTAATC; NotI_BRAF-FL forward, GGGGGCGGCCGCACCATGGCGGCGCTGAGCG) and cloned into RCASBP(A) plasmid. BRAF plasmids were provided by N. Rosen (Memorial Sloan-Kettering Cancer Center, New York, New York, USA).

Tumor induction. Neonatal Ntv-a mice were injected with $1 \mu \mathrm{l}$ suspension containing approximately 40,000 virus-producing DF- 1 cells using a $10-\mu 1$ Hamilton syringe. After injection, mice were monitored for neurological symptoms. Mice were sacrificed, and brain tissue was fixed in $10 \%$ buffered formalin. All animal experiments were conducted according to the guidelines of the ATBW (authorities for animal welfare) and were approved by the Regierungspräsidium Karlsruhe (G-18/09; Karlsruhe, Germany).

Immunohistochemistry. Sections from formalin-fixed, paraffin-embedded mouse brains were stained with $\mathrm{H} \& \mathrm{E}$ or with antibodies directed against FLAG (Sigma-Aldrich), GFAP (Dako), Ki67 (Thermo Scientific), pErk (Cell Signaling), and nestin (Chemicon) on a Ventana BenchMark XT immunostainer (Ventana Medical Systems). Antigen detection was done using a DAB Detection Kit (Ventana Medical Systems).

Imaging. MRI was performed on a 9.4 Tesla experimental MR scanner (Bruker Biospec 94/20 USR) using a 4-channel surface coil as receive RF coil. Before the start of the examination, $0.5 \mathrm{mmol} / \mathrm{kg}$ body weight of a paramagnetic contrast agent (gadodiamide) was administered by intraperitoneal injection. In each animal, we performed a 3D T1-weighted fast low-angle shot (FLASH) sequence (TR, $50 \mathrm{~ms}$; TE, $1.6 \mathrm{~ms}$; pulse angle, 29.5; field of vision, $20 \mathrm{~mm} \times 20 \mathrm{~mm} \times 10 \mathrm{~mm}$; matrix, $256 \times 256 \times 40$ ). Slice direction (axial) and the center of the slice stack was chosen to be identical for all sequences.

Western blot. Protein extracts from astrocytes were subjected to electrophoresis and transferred onto a PVDF membrane for immunoblot analysis. The following antibodies were used: BRAF-C19 (1:5,000; Santa Cruz), pErk1/2 (1:1,000; Cell Signaling), Erk1/2 (1:1,000; Cell Signaling), GAPDH (1:1,000; Calbiochem).

Quantitative RT-PCR. Real-time PCR for BRAF and 3 housekeeping genes was performed on an ABI Prism 7900 using the following primers: BRAF forward, CTCGAGTGATGATTGGGAGATTCCTGATGG; BRAF reverse, CTGCTGAGGTGTAGGTGCTGTCAC; Lmna forward, CAGCAGTCTCGAATCCGCA; Lmna reverse, AGGTCACGCAGCTTTGCCT; Ldha forward, GAAGGACTTGGCGGATGAG; Ldha reverse, CCGCGGTGATAAT-
GACCAG; Abcd1 forward, AGTGCATCTGGCCTGCTCAT; Abcd1 reverse, CTGTACGCTCACTAACCAGC.

In vitro transduction. Neurospheres from neonatal mice were cultivated in medium consisting of Neurobasal A (Gibco, Invitrogen), B27 serum-free supplement (Gibco, Invitrogen), heparin (100 $\mu \mathrm{g} / \mathrm{ml}$, Sigma-Aldrich), and glutamine. For in vitro transduction, neurospheres from Ntv-a mice were dissociated with trypsin and resuspended in serum-containing conditioned medium from virus-producing DF-1 cells. Under these conditions, cells undergo astrocytic differentiation. Infection was repeated 3 times. Cells were treated with $5 \mu \mathrm{M}$ sorafenib (provided by Bayer-Schering Pharma) or DMSO 48 hours prior to analysis.

Flow cytometry. Cell proliferation was analyzed with the Click-iT EdU assay (Invitrogen) according to the manufacturer's protocol. In brief, cells were incubated with $10 \mu \mathrm{M}$ EdU for 48 hours before measurement. Incorporated EdU was detected by conjugation of Alexa Fluor 488 and subsequent flow cytometry on FACS Canto (BD Biosciences). For each measurement, at least 10,000 events were counted. Data were analyzed using FACS Diva software (BD Biosciences).

Statistics. For statistical analysis, 2-tailed Student's $t$ test was used. $P$ values of 0.05 or less were considered significant.

\section{Acknowledgments}

We thank Neal Rosen for providing us with BRAF plasmids. This work was supported by grants from the German Federal Ministry of Education and Research (BMBF) within the National Genome Research Network NGFNplus (01GS0883) as well as by funds from the Sibylle-Assmus Award 2009 to S. Pfister. J. Gronych and J. Bageritz have been supported by the Helmholtz International Graduate School for Cancer Research.

Received for publication August 6, 2010, and accepted in revised form January 19, 2011.

Address correspondence to: Peter Lichter or Stefan Pfister, German Cancer Research Center, Im Neuenheimer Feld 280, D-69120 Heidelberg, Germany. Phone: 49.6221.42.4593; Fax: 49.62.21.42.4639; E-mail: peter.lichter@dkfz.de (P. Lichter). Phone: 49.6221.42.4618; Fax: 49.62.21.42.4639; E-mail: s.pfister@dkfz.de (S. Pfister).
1. World Health Organization, and International Agency for Research on Cancer. WHO Classification of Tumours of the Central Nervous System. 4th ed. Lyon: International Agency for Research on Cancer; 2007.

2. Dirven CM, MooijJJ, Molenaar WM. Cerebellar pilocytic astrocytoma: a treatment protocol based upon analysis of 73 cases and a review of the literature. Childs Nerv Syst. 1997;13(1):17-23.

3. Pfister S, Witt O. Pediatric gliomas. Recent Results Cancer Res. 2009;171:67-81.

4. Pfister $\mathrm{S}$, et al. BRAF gene duplication constitutes a mechanism of MAPK pathway activation in low-grade astrocytomas. J Clin Invest. 2008;118(5):1739-1749.

5. Forshew T, et al. Activation of the ERK/MAPK pathway: a signature genetic defect in posterior fossa pilocytic astrocytomas. J Pathol. 2009;218(2):172-181.

6. Jones DTW, et al. Tandem duplication producing a novel oncogenic BRAF fusion gene defines the majority of pilocytic astrocytomas. Cancer Res. 2008;68(21):8673-8677.

7. Jones DTW, Kocialkowski S, Liu L, Pearson DM, Ichimura K, Collins VP. Oncogenic RAF1 rearrangement and a novel BRAF mutation as alternatives to KIAA1549:BRAF fusion in activating the
MAPK pathway in pilocytic astrocytoma. Oncogene. 2009;28(20):2119-2123.

8. Sievert A, et al. Duplication of $7 \mathrm{q} 34$ in pediatric low-grade astrocytomas detected by high-density single-nucleotide polymorphism-based genotype arrays results in a novel BRAF fusion gene. Brain Pathol. 2009;19(3):449-458.

9. Jacob K, et al. Duplication of $7 \mathrm{q} 34$ is specific to juvenile pilocytic astrocytomas and a hallmark of cerebellar and optic pathway tumours. Br J Cancer. 2009;101(4):722-733.

10. Lyustikman Y, Momota H, Pao W, Holland EC. Constitutive activation of Raf- 1 induces glioma formation in mice. Neoplasia. 2008;10(5):501-510.

11. Uhrbom L, Dai C, Celestino JC, Rosenblum MK, Fuller GN, Holland EC. Ink4a-Arf loss cooperates with KRas activation in astrocytes and neural progenitors to generate glioblastomas of various morphologies depending on activated Akt. Cancer Res. 2002; 62(19):5551-5558.

12. Uhrbom L, Kastemar M, Johansson FK, Westermark B, Holland EC. Cell type-specific tumor suppression by Ink4a and Arf in Kras-induced mouse gliomagenesis. Cancer Res. 2005;65(6):2065-2069.

13. Dai C, Celestino JC, Okada Y, Louis DN, Fuller
GN, Holland EC. PDGF autocrine stimulation dedifferentiates cultured astrocytes and induces oligodendrogliomas and oligoastrocytomas from neural progenitors and astrocytes in vivo. Genes Dev. 2001;15(15):1913-1925.

14. Hambardzumyan D, Amankulor NM, Helmy KY, Becher OJ, Holland EC. Modeling adult gliomas using RCAS/t-va technology. Transl Oncol. 2009;2(2):89-95.

15. Shih AH, Dai C, Hu X, Rosenblum MK, Koutcher JA, Holland EC. Dose-dependent effects of platelet-derived growth factor-B on glial tumorigenesis. Cancer Res. 2004;64(14):4783-4789.

16. Robinson JP, VanBrocklin MW, Guilbeault AR, Signorelli DL, Brandner S, Holmen SL. Activated BRAF induces gliomas in mice when combined with Ink4a/Arf loss or Akt activation. Oncogene. 2010;29(3):335-344.

17. Pritchard $\mathrm{C}$, et al. Mouse models for BRAFinduced cancers. Biochem Soc Trans. 2007; 35(pt 5):1329-1333.

18. Grbovic OM, et al. V600E B-Raf requires the Hsp90 chaperone for stability and is degraded in response to Hsp90 inhibitors. Proc Natl Acad Sci U S A. 2006;103(1):57-62. 